Revue européenne des sciences sociales

European Journal of Social Sciences

52-1 | 2014

Politiques du libre accès en sciences humaines et sociales

\title{
Les enjeux normatifs et politiques de la diffusion de la recherche
}

Bénéfices sociaux et libre accès

\section{Xavier Landes}

\section{OpenEdition \\ Journals}

Édition électronique

URL : http://journals.openedition.org/ress/2663

DOI : $10.4000 /$ ress.2663

ISSN : $1663-4446$

Éditeur

Librairie Droz

Édition imprimée

Date de publication : 6 mai 2014

Pagination : 65-92

ISBN : 978-2-600-01829-6

ISSN : 0048-8046

\section{Référence électronique}

Xavier Landes, "Les enjeux normatifs et politiques de la diffusion de la recherche ", Revue européenne des sciences sociales [En ligne], 52-1 | 2014, mis en ligne le 12 mai 2014, consulté le 04 mai 2019. URL : http://journals.openedition.org/ress/2663 ; DOI : 10.4000/ress.2663 


\title{
LES ENJEUX NORMATIFS ET POLITIQUES DE LA DIFFUSION DE LA RECHERCHE BÉNÉFICES SOCIAUX ET LIBRE ACCÈS
}

XAVIER LANDES

Université de Copenhague xavier.landes@gmail.com

Résumé. Le savoir est une activité coopérative essentielle pour les sociétés industrielles. Base de leur modèle économique, il produit par ailleurs de nombreux bénéfices matériels, socio-politiques et distants, en particulier au travers de sa diffusion. Dans ce contexte, il devient important de déterminer les principes qui devraient orienter la répartition des coûts qu'une telle diffusion implique. La Recommandation de la Commission européenne du 17 juillet 2012 va dans ce sens en proposant de rendre gratuit l'accès aux résultats des recherches financées par des fonds publics. Elle offre ainsi un cadre idéal pour discuter de la juste répartition des coûts de diffusion du savoir.

Mots-clés: bénéfices sociaux, État, libre accès, publications académiques, recherche.

\begin{abstract}
Knowledge is a cooperative activity, which is essential to industrial societies. Basis of their economic model, it also produces numerous material, socio-political and distant benefits. In this context, it becomes important to determine the principles that should orientate the reparation of the costs of knowledge diffusion. The Recommendation of the European Commission of July $17^{\text {th }} 2012$ goes in this direction by proposing to make free the access to research results financed by public funds. It then offers an ideal framework for discussing the just repartition of the costs of knowledge diffusion.
\end{abstract}

Keywords: academic publishing, open access, research, social benefit, State. 
Le savoir est au cœur des sociétés industrielles. Depuis le XVIII siècle, sa production et son accumulation ont été de puissants moteurs de développement économique et social (Persson, 2010). Le savoir constitue un des domaines de prédilection de la coopération humaine (Heath, 2006, p. 327-330) qui implique la contribution de divers acteurs tels que chercheurs, organismes de recherche, bailleurs de fonds, maisons d'édition, etc. L'émergence d'économies de l'information a renforcé cette importance (Cohen, 2006), comme en témoigne le débat relatif aux droits de propriété intellectuelle et artistique sur Internet.

Les sociétés industrielles étant fondées sur la spécialisation et la division du travail, cela implique que certaines institutions ont pour fonction de produire, aider à la production ou diffuser le savoir. Les institutions publiques jouent un rôle central dans ces activités, en particulier au travers des universités et établissements de recherche financés par l'impôt. Le savoir n’est toutefois pas l'apanage des seuls États. Des organisations privées (universités, fondations, entreprises, maisons d'édition, etc.) contribuent aussi à l'économie du savoir.

Dans ce contexte, le savoir suscite de plus en plus d'attention et de débats quant aux mécanismes institutionnels qui président à sa production et à sa diffusion. En guise d'illustration, un débat fait rage depuis quelques années à propos de certaines maisons d'édition accusées de profiter de la recherche, principalement publique, afin d'extraire de confortables profits, à l'image des géants du secteur organisés en quasi-oligopole tels Elsevier, Springer ou WileyBlackwell (Collectif, 20I3c). Le reproche principal est que ces entreprises, aux profits colossaux, tireraient un avantage indu de l'investissement fait par d’autres acteurs, notamment publics (Landes et al., 20I3b). Ce débat porte sur la répartition des charges et bénéfices de la production et de la diffusion du savoir en tant qu'activités de coopération. En d'autres termes, les objections adressées aux maisons d'édition ainsi que les arguments invoqués par ces dernières tournent autour de la question suivante: comment les coûts et bénéfices de la diffusion du savoir doivent-ils être répartis entre les divers acteurs du domaine?

I Depuis 2012, l'initiative The Cost of Knowledge incite les chercheurs à boycotter le leader de la publication académique (Elsevier), c'est-à-dire à refuser de publier, évaluer ou éditer des textes pour une des revues du groupe:<http://thecostofknowledge.com>. 
La Recommandation de la Commission européenne (CE) du I7 juillet 2012 doit être interprétée dans cette perspective. [Voir l'article d'Ivan Jaffrin et Thomas Parisot, dans le présent dossier, qui rend compte des débats suscités en France par la Recommandation.] En mettant l'accent sur la libre accessibilité des résultats de la recherche financée sur fonds publics, la $\mathrm{CE}$ adopte une certaine position sur la question de la répartition des coûts de la diffusion d'une recherche financée par des fonds publics. L’exécutif européen esquisse ainsi les termes généraux de la coopération humaine appliquée au savoir.

Le but de cet article est de dégager un certain nombre d'enjeux, principalement normatifs, soulevés par la diffusion de la recherche et de situer la Recommandation dans ce cadre. Cet objectif est poursuivi tout au long de quatre sections. La première identifie les différents acteurs liés à la diffusion de la recherche ainsi que de leurs intérêts. La deuxième présente les bénéfices sociaux liés à la recherche et les ordonne en trois catégories : matériels, socio-politiques et distants. La troisième discute de la répartition des charges de la diffusion de la recherche tout en avançant le principe d'une implication de l'État. La quatrième fournit une rapide analyse critique du contenu de la Recommandation tandis que la conclusion dessine les contours de quelques questions fondamentales liées au passage à l'Open Access (OA) ou libre accès².

\section{PLURALITÉ DES INTÉRÊTS LIÉS À LA DIFFUSION DE LA RECHERCHE}

La recherche, notamment en sciences humaines et sociales (SHS), implique une diversité d'acteurs qui poursuivent des buts qui convergent, divergent ou s'opposent. Suivant une dénomination issue de l'éthique des affaires, ces acteurs sont les «parties prenantes » de la recherche, c'est-à-dire de la création du savoir, dans le sens où leurs intérêts sont affectés par celle-ci. Lors de la conduite ou de la diffusion de la recherche, ces acteurs poursuivent des objectifs qui entrent en résonnance ou en conflit. Les principaux acteurs sont donc a) les chercheurs, b) les institutions de recherche, c) les bailleurs de fonds, d) les diffuseurs et e) les destinataires de la recherche.

2 Dans ce texte, Open Access $(\mathrm{OA})$, libre accès et accès libre sont alternativement utilisés afin de caractériser la même chose: la mise à disposition gracieuse des fruits de la recherche. 
a) Les chercheurs ont pour activité principale, mais non unique (enseignement par exemple), de produire du savoir. Ils ont été formés dans ce but, souvent au sein d'établissements publics. Leur intérêt est de produire un savoir pertinent, c'est-à-dire reconnu comme tel par des pairs issus de leur discipline (Pedersen and Stjernfelt, 2014). Cependant, du fait de l'importance croissante du nombre de publications pour l'avancement de leur carrière (pour l'obtention d'un poste, d'une promotion ou de fonds de recherche), de plus en plus de chercheurs donnent la priorité à la quantité au détriment de leur qualité (que l'on peut évaluer en termes de contribution à l'avancée du savoir ou de retombées plus larges pour la société dans son ensemble) (Landes et al., 2011, 2013a).

b) Les institutions de recherche rassemblent universités et d'autres organismes spécialisés (comme le CNRS en France) dont l'objectif premier est proposer une recherche de qualité. De ce point de vue, l'émergence des classements internationaux d'universités comme celui de l'université de Shanghai ${ }^{3}$ ou le Times Higher Education World University Ranking ${ }^{4}$ a conduit de plus en plus d'établissements à miser sur des disciplines à fort potentiel de visibilité et d'attractivité (pour attirer des fonds, chercheurs et étudiants) ${ }^{5}$, sur les sciences dites «dures » au détriment des sciences dites «molles» (car les premières se prêtent plus aux applications industrielles et partenariats privé-public) ou sur les disciplines appliquées au détriment de celles fondamentales dont les résultats ne sont pas exploitables dans l'immédiat ${ }^{6}$. Les établissements de recherche s'emploient à identifier les secteurs porteurs en termes de brevets ou de publications afin d'y concentrer leurs efforts. Cette tendance est accentuée par la mise en place du «nouveau management public » qui met l'accent sur l'évaluation quantifiée des résultats de recherche (Newfield, 2011 ; Pollitt, 1993 ; Weiler, 2000).

3 Voir <http://www.shanghairanking.com>.

4 Voir<http://www.timeshighereducation.co.uk/world-university-rankings/2012-I3/world-ranking>.

5 Ce qui génère des «bulles» de publications comparables aux bulles spéculatives dans certaines disciplines (Pedersen and Hendricks, 2013).

6 La France est en bonne position quant à la part de la recherche fondamentale dans l'ensemble des dépenses de recherche (Cour des comptes, 2013, p. 162). 
c) Dans le cas qui nous intéresse, c'est-à-dire celui de la Recommandation, la catégorie des bailleurs de fonds englobe des bailleurs de fonds publics tels que le Conseil danois pour la recherche indépendante ${ }^{7}$. L'une des raisons d'être de ces institutions est de mettre en concurrence les chercheurs ou équipes de recherche pour leur financement. La justification est que la concurrence est un puissant émulateur qui accroît la qualité de la recherche. L’intérêt des bailleurs de fonds est donc de procéder à une allocation optimale des ressources qui leur sont imparties, c'est-à-dire de financer les meilleurs projets. Le «nouveau management public » a aussi un effet sur cette catégorie d'acteurs au travers de l'évaluation des procédures d'octroi de fonds de recherche.

d) La quatrième catégorie est celle des diffuseurs de la recherche. Ce terme générique regroupe des acteurs qui servent de relais entre les producteurs de savoir et un public plus large, incluant spécialistes et non-spécialistes. Il sagit en général de maisons d'édition. Ce secteur a connu depuis les années 1990 une double révolution.

D’une part, les nouvelles technologies de l'information ont diminué les coûts d'administration des revues scientifiques, ce qui a conduit à l'augmentation de leur nombre. L'une des conséquences a été l'apparition et le développement des diffuseurs en OA, c'est-à-dire dont le contenu est d'accès libre ${ }^{8}$. In fine, les chercheurs ont accès à un nombre sans cesse croissant de revues, journaux et autres publications afin de diffuser leurs résultats.

D’autre part, et de manière paradoxale, un phénomène de concentration a eu lieu, donnant naissance, surtout dans le monde anglo-saxon, à de véritables multinationales de la publication scientifique comme Elsevier, Wiley-Blackwell ou Springer aux taux de marge records (30\% ou plus du chiffre d’affaires). Ces géants sont accusés d’avoir profité d'une entente implicite, qui existait avant la double révolution, entre éditeurs et chercheurs (Guterson, 2012). Les premiers, dont la rentabilité était en général faible et qui étaient principalement des éditeurs universitaires

7 Voir<http://fivu.dk/en/research-and-innovation/councils-and-commissions/the-danishcouncil-for-independent-research>.

8 II existe deux formes d'OA. Le premier est l'accès en or (Gold $\mathrm{OA}$ ) dans lequel la publication elle-même est d'accès gratuit. Le second est l'accès vert (Green $O A$ ) dans lequel l'auteur archive une version de son article qui est accessible (et non la version publiée). 
ne cherchant pas à faire du profit, diffusaient la production des seconds en échange d'un travail gracieux (pas de rémunération pour la publication, l'évaluation de la qualité scientifique des textes ou le travail éditorial). Ainsi les chercheurs offraient leur travail aux éditeurs afin que ces derniers puissent diffuser leurs recherches.

Certains éditeurs sont accusés d’avoir profité de cette entente afin d’extraire des profits importants, cristallisant ainsi l'opposition des chercheurs, institutions de recherche et, chose nouvelle, des bailleurs de fonds (ce qu'exprime en filigrane le texte de la Recommandation). Toutefois, il existe d'autres diffuseurs plus «traditionnels» au modèle économique précaire dont les recettes couvrent à peine les dépenses de fonctionnement. Le cœur de métier de ces diffuseurs est en général (mais pas tout le temps) la version papier de leurs revues, la version électronique n'ayant été développée que sur le tard et en complément. De nombreuses revues de SHS en langue française se trouvent dans cette catégorie ${ }^{9}$.

Quoi qu'il en soit, la plupart des éditeurs cherchent à sécuriser des revenus suffisants pour maintenir leurs activités. En tant que diffuseurs du savoir, ils prennent en charge la sélection, la mise en forme ainsi que l’accessibilité (sous une forme matérielle ou virtuelle) des résultats de la recherche. En d’autres termes, ils transforment une matière première - les articles qui leur sont soumis à évaluation - en produits finis - les articles corrigés, mis en forme et organisés dans des revues. En outre, certains diffuseurs offrent également le prestige ainsi que le gage de sérieux attachés à leur nom. [À ce propos, se référer à l'article d'Olivier Bomsel dans le présent dossier.]

e) La dernière catégorie est celle des destinataires de la recherche. Elle regroupe une grande diversité d’acteurs incluant les entreprises qui mettent en place des procédures ou produits innovants, les citoyens qui veulent mieux comprendre le monde ou informer leur action et les autres chercheurs qui utilisent les résultats de leurs pairs afin de mener leurs propres recherches. Cette catégorie rassemble de manière générale des acteurs qui ont tout intérêt à ce que la recherche soit accessible avec le moins d'entraves possible.

9 Une troisième catégorie peut être identifiée. Elle inclut les revues dont la base est virtuelle dans le sens où elles publient uniquement ou avant tout en ligne. C'est dans ce dernier groupe que se trouvent les principaux acteurs du mouvement de l'OA. 
Ce bref aperçu permet de cerner les enjeux en présence. Par exemple, on comprend mieux les raisons pour lesquelles une course à la publication s'est mise en branle lors des deux dernières décennies : car l'intérêt des chercheurs, institutions de recherche, bailleurs de fonds et diffuseurs va, apparemment, en ce sens. S'expliquent aussi les tensions actuelles quant au mode d'accès à la recherche. Alors qu'un accès libre (tel que préconisé par exemple par la Recommandation) est la solution vers laquelle inclinent naturellement les chercheurs, bénéficiaires de la recherche, voire les institutions de recherche et les bailleurs de fonds, les diffuseurs de la recherche ont un intérêt à maintenir un accès payant.

Bien qu'incomplète une telle grille de lecture fournit une base intéressante pour quiconque veut se livrer à une sociologie ou une économie de la recherche et de sa diffusion. Il est important de garder à l'esprit cette pluralité d'acteurs et d'intérêts lorsque l'on identifie les attentes que la société peut légitimement nourrir à l'égard de la diffusion de la recherche.

\section{LES BÉNÉFICES SOCIAUX DE LA RECHERCHE}

Considérant les mécanismes coopératifs - ou compétitifs - au sein de nos sociétés, il est bon de s'interroger sur la fonction que remplit ledit mécanisme ainsi que sur les attentes légitimes à son égard. De ce point de vue, la recherche constitue la colonne vertébrale des sociétés industrielles. En effet, ces dernières s'appuient sur l'innovation afin de garantir croissance économique et amélioration de la qualité de vie (qui ne vont souvent pas de pair, comme le démontre la littérature critique du productivisme contemporain [Méda, 2008]).

Avant de poursuivre, il est nécessaire de faire une clarification. Dans les discussions sur la recherche, il est de coutume de distinguer les recherches «utilitaires » dans le sens où elles apporteraient un gain concret de celles «non utilitaires » en ce qu'elles ne promettraient pas un tel type de gain. La distinction sert en général à souligner la spécificité des SHS qui ne pourraient pas être soumises à un objectif de rentabilité (pour le meilleur comme pour le pire) et qui appartiendraient donc à la seconde catégorie. La distinction est toutefois caricaturale en ce qu'elle donne à penser qu'il y aurait d'un côté la recherche 
qui apporterait «quelque chose» à la société et de l'autre côté la recherche purement gratuite, entreprise pour le pur plaisir de chercher.

Il est toutefois difficile de considérer que l'égyptologie, la philosophie, la littérature comparée, l'histoire du Moyen Âge et bien d’autres domaines soient purement «gratuits» dans le sens d'inutiles, superficiels ou superflus. Chaque discipline porte en soi un gain social qui peut prend des formes diverses (et souvent imprévisibles) : des réflexions plus poussées à propos de nos institutions (philosophie politique), de la nature de nos sociétés (sociologie, anthropologie), des civilisations qui nous ont précédés (histoire) ou de celles qui coexistent avec nous (ethnologie), etc. En ce sens, la recherche en SHS nourrit la connaissance que nous avons de nous-mêmes en tant qu'êtres humains, de nos histoires, de nos modes d'organisation, de nos productions matérielles et symboliques. Elle nous offre ainsi une compréhension plus fine et informée du fait humain. Sauf à considérer cette somme de savoirs comme totalement vaine, il est difficile de ne pas reconnaître que la recherche contribue de manière positive à la qualité de nos vies, c'est-à-dire qu’elle produit des «gains», quelle que soit la compréhension que l'on peut avoir de tels gains.

Plusieurs types de bénéfices sociaux sont attribués à la recherche, notamment en SHS ainsi qu'à sa diffusion. Dans les textes internationaux, il est courant de trouver des références à l'innovation, aux futures découvertes qui découleraient d'une recherche donnée, aux gains d'efficacité, à la production d’externalités positives. Ces bénéfices peuvent être classés en trois catégories : a) bénéfices matériels, b) bénéfices socio-politiques, et c) bénéfices distants.

a) Bénéfices matériels. Tout d’abord, la recherche est supposée accroître l'efficience des sociétés industrialisées définie comme la capacité de faire mieux avec autant ou aussi bien avec moins tout en améliorant la situation des membres de ces sociétés ${ }^{10}$. De ce point de vue, l'efficience possède une dimension industrielle et commerciale très claire. En effet, la recherche est utile pour développer des idées, concepts, systèmes, procédures, biens, services qui permettent d’accroître la

10 Pour une discussion détaillée du concept d'efficience, le lecteur peut se reporter à l'ouvrage didactique de Joseph Heath, La Société efficiente (2002 [200 I]). 
productivité humaine et l'efficacité de nos institutions. Cette dimension explique à la fois l'emphase mise sur la recherche appliquée, notamment au détriment de la recherche fondamentale (voir supra), et certains choix de financement.

Ce rôle du savoir comme instrument de l'amélioration des conditions matérielles de l'humanité est évident depuis la révolution néolithique. Au cœur de la production du savoir et de sa diffusion se loge une dynamique puissante de remplacement des connaissances surannées et d'amélioration de celles existantes inhérente aux sociétés industrielles qui doivent leur vitalité à un processus de «destruction créatrice» (Schumpeter, 1990 [1942]). Au-delà de la dimension strictement matérielle, la production et la diffusion du savoir concourent aussi à l'amélioration des conditions de vie. Ce bénéfice est évident dans des domaines comme la médecine. Ce n'est pas un hasard si l'espérance de vie a triplé depuis 1740 en France ${ }^{11}$. Ce bénéfice, parmi d’autres, est le résultat direct de la production et de la diffusion du savoir.

Preuve supplémentaire de l'importance du savoir et de la complexité de ses enjeux, les attentes qu'expriment la société ainsi que les organisations internationales comme l'UNESCO (Projet révisé de stratégie relatif à la contribution de l'UNESCO à la promotion de l'accès libre à l'information et à la recherche scientifiques, 20I I) ou l'OCDE (Déclaration sur l'accès aux données de la recherche financée par des fonds publics, 2004) vont au-delà de cette simple vision productiviste. Il est attendu du savoir des bénéfices qui dépassent l’amélioration des standards de vie.

b) Bénéfices socio-politiques. Le savoir génère des effets sociaux et politiques positifs sous la forme d'empowerment, c'est-à-dire d'une maitrise accrue des citoyens sur leur existence. Il s'agit de l'un des principaux bénéfices de la recherche en SHS. De ce point de vue, la diffusion du savoir a des effets variables, voire opposés, en fonction de ses conditions d’accessibilité. Si l'on se penche sur la littérature fournie par les organisations internationales, un savoir accessible génère des bénéfices d’un double point de vue : inclusion et égalité.

II Voir Institut National des Études Démographiques: <http://www.ined.fr/fr/tout_savoir_population/graphiques_mois/esperance_vie_france/>. 
L'inclusion des individus au sein des sociétés est un bénéfice de la diffusion des résultats de la recherche (Décision I87 EX/ıo de l’UNESCO). Le savoir permet aux individus de développer des compétences, se fixer des buts et les poursuivre, mener une carrière professionnelle épanouissante, etc. Le savoir est aussi un «capital» qui peut être un objet de luttes sociales autant qu'un instrument d'exclusion et de domination (Bourdieu, Passeron, I970; Bourdieu, 1979).

L'égalité est un autre bénéfice du libre accès à la recherche, et ceci sous plusieurs formes. Tout d'abord, l'égalité de participation est intimement liée au savoir et à sa diffusion. En effet, la connaissance constitue le prélude indispensable à toute action informée des citoyens, a fortiori dans une société de l'information. Cette importance est reconnue par de nombreux textes internationaux à l'instar de la Déclaration universelle des droits de l'homme ${ }^{12}$ ou du Pacte international relatif aux droits économiques, sociaux et culturels ${ }^{13}$. En 20r2, l'ALLEA (ALL European Academies), qui représente l'ensemble des Académies des sciences et humanités en Europe, a produit une déclaration en faveur d'une science ouverte qui s'appuie, entre autres, sur l'égalité de participation ${ }^{14}$.

Ensuite, le savoir est censé favoriser une autre forme d'égalité : l'égalité d’opportunités. Dans des sociétés complexes, l'accès au savoir peut se révéler crucial pour les individus afin qu'ils puissent poursuivre leurs plans de vie de manière informée et efficace. Il n’est guère contestable que l’accès au savoir ainsi que la maîtrise de certains codes qui y sont associés constituent une source majeure

12 L'article 27, alinéa I, stipule que «toute personne a le droit de prendre part librement à la vie culturelle de la communauté, de jouir des arts et de participer au progrès scientifique et aux bienfaits qui en résultent».

13 L'article 15, alinéa I, affirme que «les États parties au présent Pacte reconnaissent à chacun le droit: a) de participer à la vie culturelle; b) de bénéficier du progrès scientifique et de ses applications; c) de bénéficier de la protection des intérêts moraux et matériels découlant de toute production scientifique, littéraire ou artistique dont il est l'auteur». L'alinéa 2 poursuit de la manière suivante: «Les mesures que les États parties au présent Pacte prendront en vue d'assurer le plein exercice de ce droit devront comprendre celles qui sont nécessaires pour assurer le maintien, le développement et la diffusion de la science et de la culture».

14 Voir<http://www.unesco.org/new/en/communication-and-information/resources/newsand-in-focus-articles/all-news/news/open_science_for_the_2lst_century_declaration_of_ all_european_academies/\#.UsqNUnkkdb4>. 
d'inégalités d'opportunités parmi les citoyens. Les derniers résultats de l'enquête PISA confirment cette importance (Battaglia, Collas, 20I3).

Le savoir tient une place particulière dans les sociétés modernes. D’une part, il fut au cœur de la Révolution industrielle ainsi que des avancées techniques et technologiques qui ont suivi. D’autre part, il a été (et, dans une certaine mesure, est toujours) considéré comme la pierre angulaire dans la formation du citoyen cultivé, éclairé et autonome ${ }^{15}$. L'insistance de divers acteurs (à l'instar des organisations internationales telles que l'ONU, l'UNESCO ou l'OCDE) sur les principes d'égalité et d'inclusion comme déterminant l'accès au savoir, mais aussi comme en caractérisant les bénéfices socio-politiques, souligne le caractère central du savoir dans la formation du citoyen moderne.

c) Bénéfices distants. Dans la littérature, il est courant d’identifier des bénéfices distants à la recherche et à sa diffusion (Salter, Martin 2001). Ceux-ci correspondent en partie à ce que les économistes qualifient d'« externalités positives », c'est-à-dire des avantages dont un agent profite sans rémunérer le producteur de tels avantages ${ }^{16}$. En ce sens, la recherche profite indirectement à une multitude d'acteurs, au travers d'avancées technologiques par exemple. Parmi les effets distants, il est possible de classer une vie démocratique de qualité et stimulante. L'argument peut prendre la forme suivante : plus le savoir est accessible, plus le niveau des débats publics ainsi que la qualité des décisions et politiques s’améliorent. La difficulté de taille est que de tels effets sont en général très difficiles à évaluer, précisément en raison de leur caractère distant et diffus.

15 Cette importance est visible dans l'idéal républicain français basé sur le modelage d'un citoyen dont l'acquisition du savoir (incarné par une solide culture générale) est le vecteur d'entrée dans la culture (par opposition à la nature ou à la barbarie). Ce qui explique l'importance particulière attribuée à l'école.

I6 L'exemple canonique dans la littérature économique est celui du phare. En effet, le phare produit de la lumière pour tous les bateaux qui croisent dans les environs, même pour ceux qui ne paient pas pour ce service. 
En résumé, la diffusion de la recherche produit des bénéfices dont l'ensemble de la société profite, et ceci à plusieurs niveaux. Les diffuseurs jouent un rôle prépondérant dans le sens où ils servent d'interfaces entre les chercheurs et un public plus large. L’accessibilité des résultats de la recherche a donc un impact direct sur le type ainsi que l'étendue des bénéfices sociaux de la recherche. L'importance de la diffusion soulève la question, centrale, de la répartition des charges qui y sont liées.

\section{LA RÉPARTITION DES CHARGES LIÉES À LA DIFFUSION DE LA RECHERCHE}

Avant de discuter de la Recommandation de la CE en tant que telle, il convient d'exposer la situation actuelle ainsi que quelques arrangements alternatifs. Ce faisant, il est important de garder à l'esprit que cette section dessine une tendance générale quant à la diffusion de la recherche en SHS, tendance générale inspirée par le monde anglo-saxon. L'importance de ce dernier se comprend par la primauté de la recherche en langue anglaise. De plus, au sein de la recherche anglo-saxonne se profile un véritable modèle économique - concentré, oligopolistique - qui mérite d'être débattu dans la perspective de la Recommandation de la CE.

Comme de nombreux chercheurs le dénoncent, le modèle qui est en train de s'imposer est fondé sur une rente de situation ${ }^{17}$ dont bénéficient les principales maisons d'édition, plus particulièrement anglo-saxonnes (Landes et al., 20ızb). Des universités, en majorité financées par des fonds publics (c'est-à-dire par l'impôt), ainsi que des bailleurs de fonds, souvent publics (financés aussi par l'impôt), paient des universitaires pour qu'ils mènent des recherches, présentent leurs résultats, discutent ceux des autres, écrivent des articles, les mettent en forme et les envoient à des diffuseurs.

17 Une rente de situation peut être définie comme une situation dans laquelle un agent extraie des bénéfices constants et indus du fait de la manière dont l'institution ou les relations dont il fait partie sont structurées. L'archétype de la rente de situation est le monopole dans lequel une entreprise peut imposer le prix qu'elle souhaite et donc extraire un revenu au-dessus de ses coûts de revient et supérieur à celui qui serait le sien dans un équilibre concurrentiel. 
Nombre de chercheurs reprochent le fait que des fonds publics servent à payer les chercheurs pour produire du savoir, l'évaluer à fin de publication, mettre les textes en forme sans que les résultats soient pour autant librement accessibles (Conley, Wooders, 2009). En effet, les universités, bibliothèques publiques et citoyens doivent payer pour accéder aux résultats de la recherche. Souvent ces sommes sont significatives. Pour le dire autrement, il y a le sentiment que la «société », c'est-à-dire les citoyens au travers des institutions publiques, paie plusieurs fois pour le même produit (Aberganti, 20I3). C'est le problème auquel la Recommandation de la CE entend répondre en défendant le principe de l'OA pour la recherche financée sur fonds publics.

Mais avant de discuter de la Recommandation en tant que telle, deux points méritent d'être abordés. Dans un premier temps, il est nécessaire de se demander s'il n'est pas possible de trouver des justifications au système actuel, c'est-à-dire au principe d'une facturation imposée aux lecteurs finaux, que ces derniers soient des individus ou des institutions (universités et bibliothèques). En effet, il est possible de juger que bien que les chercheurs, bailleurs de fonds et institutions de recherche souhaitent que la recherche soit accessible au plus grand nombre, ils n'expriment que leurs propres intérêts : les chercheurs veulent bénéficier de l'audience que leur conférerait une diffusion libre de leurs travaux, les institutions de recherche pourraient plus aisément prouver leur valeur auprès des institutions de tutelle ou attirer de nouveaux étudiants tandis que les bailleurs de fonds auraient le sentiment de maximiser la rentabilité de leurs investissements. Pareils intérêts demeurent des intérêts particuliers dont ni la légitimité ni la faisabilité ne sont garanties a priori.

Dans un second temps, il est nécessaire de considérer d’autres arrangements quant à la répartition des charges inhérentes à la diffusion de la recherche ainsi que les principes qui pourraient inspirer de tels arrangements. Le but est de construire une grille d'analyse, un tamis normatif au travers duquel on pourrait juger de la juste répartition des charges et bénéfices de la recherche académique. 
Concernant la première question - la justification du système actuel -, une possibilité est de considérer que les diffuseurs offrent un service réel qui doit être rétribué. En effet, entre le «produit brut» fourni par le chercheur, même sous la forme d'un texte mis aux normes de la revue, et le «produit fini», c'est-à-dire l'article publié dans une revue, il y a une différence qui peut être importante. Le texte doit être relu, corrigé, mis en page. Un éditorial est parfois nécessaire pour présenter le contenu de la revue, mettre les articles en perspective et dégager une vision d'ensemble ${ }^{18}$. Donc, les chercheurs, institutions de recherche, bailleurs de fonds et citoyens peuvent bien vouloir accéder gratuitement au savoir, cela reste un vœu pieux qui fait fi des contraintes matérielles qu'une telle diffusion implique.

Contre cette défense des pratiques des éditeurs, deux critiques sont envisageables. La première consiste à souligner que la «valeur ajoutée» apportée par les éditeurs est souvent absente. Par exemple, nombre de revues scientifiques offrent un service éditorial limité ou l'externalisent complètement (en faisant appel à des éditeurs «invités » qui évaluent, choisissent les textes, écrivent une introduction, s'assurent que les textes sont au format de la revue sans être rémunérés). L'éditeur de la revue (souvent le seul universitaire à être payé, quand il l'est) se limite d'habitude à prendre les décisions finales concernant les manuscrits sur la base d'évaluations fournies par des collègues non rémunérés et à contacter les auteurs. Bien souvent, aucune introduction ou éditorial n'est publié en tête de chaque livraison de la revue concernée.

Bien que cette critique paraisse fondée, il est possible de souligner que le travail éditorial limité que certaines revues fournissent ne remet nullement en question le principe d’une rémunération pour ce service, en particulier quand on considère que des revues s'acquittent de manière sérieuse de cette tâche. La légitimité d’une rémunération des diffuseurs peut être renforcée en invoquant le travail de mise en forme et de diffusion du texte (impression et production pour les versions papier, ou hébergement sur des serveurs pour les versions élec-

I8 II y a aussi le prestige conféré par certaines revues établies qui fournit une plus-value aux articles qui y sont publiés (Pedersen, Stjernfelt, 2014). 
troniques, promotion du contenu, etc.). Quel que soit le travail éditorial accompli, les diffuseurs s'acquitteraient donc d'un travail qui mériterait rémunération.

La seconde critique qui s’appuie sur la notion de mérite est une réponse à cet argument. En effet, il peut être objecté que la rémunération réclamée par les diffuseurs est en général sans rapport avec le service rendu. À la différence de la première critique, il n'est pas question de nier la valeur ajoutée fournie par les diffuseurs, mais de souligner le manque d'adéquation entre celle-ci et la rétribution demandée aux bibliothèques, universités et particuliers pour accéder au produit fini. Toutefois, hormis cas évidents de surfacturation, l'évaluation de cette critique est compliquée, car il est nécessaire d’avoir une idée du «juste prix » de la diffusion, ce qui relève d'un travail empirique d'évaluation au cas par cas.

Cette indétermination quant à la valeur ajoutée offerte par les revues explique pourquoi les diffuseurs font face à la critique d’appropriation indue d'une plusvalue : car il peut être compliqué de déterminer une rétribution qui soit «juste ». La critique porte d'autant plus que les diffuseurs peuvent être tentés de justifier les tarifs imposés par l'ensemble du «produit fini»: contenant (mise en page, travail éditorial et de diffusion), mais aussi contenu (savoir). Dans ce cas, si le prix de l’accès imposé par le diffuseur est supposé inclure la valeur du savoir en propre, alors le fait de ne pas rétribuer les chercheurs ressemble à de l'extraction de plus-value. Par ailleurs, la pratique du «Big Deal» dans lequel les maisons d'édition groupent certaines revues afin de vendre ces ensembles de manière indivise (Edlin, Rubinfeld, 2005) renforce cette suspicion à cause du manque de clarté quant au produit payé par les universités et bibliothèques.

Le cœur du problème est donc d'évaluer ce qui revient de droit aux diffuseurs, ce qui implique de calculer une répartition équitable des charges de la diffusion de la recherche. Du point de vue normatif, un tel travail suppose de déterminer des principes de répartition. En d’autres termes, quel arrangement garantit à la diffusion du savoir un impact maximal en termes d'efficience, d'inclusion et d'égalité ?

Une partie du problème se situe dans le caractère «impur» de la situation actuelle qui voit deux arrangements aux logiques différentes cohabiter, ce qui suscite incompréhensions, tensions et insatisfactions de part et d’autre. 
Le premier arrangement consiste à faire supporter les coûts de la diffusion du savoir aux seuls bénéficiaires: individus et organisations ${ }^{19}$. C'est ce mécanisme qui suscite les plus vifs débats au sein de la communauté académique. La logique de l'argument est la suivante: comme beaucoup d'agents (entreprises, individus) bénéficient de la recherche, il est légitime de leur faire payer la diffusion. L'argument s'applique d'autant plus aux institutions de recherche puisque leurs chercheurs utilisent ce même savoir pour leurs travaux, qui seront alors diffusés et discutés au nom des institutions en question, ce qui accroît in fine leur prestige.

Toutefois, cet arrangement prête le flanc à plusieurs critiques. Tout d’abord, si ce sont les utilisateurs du savoir qui doivent en supporter le coût de diffusion, il convient de spécifier quels utilisateurs sont concernés. Il devient alors possible d'adopter une conception extensive ou au contraire restrictive de la notion d'«utilisateurs ».

En vertu de la conception extensive, toute entité qui manipule le savoir entre la recherche et sa forme finale (publications) en est un utilisateur. Dans ce cas, les diffuseurs devraient également payer pour utiliser le savoir sous sa forme brute (les manuscrits) en vue de le transformer. Tout comme les entreprises achètent leurs matières premières, les éditeurs devraient aussi acheter les textes qu'ils retiennent pour publication. En l'absence de raisons convaincantes pour déplacer l'ensemble des coûts vers le consommateur final, il devient plausible d'imposer une rémunération à chaque étape de la production et de la diffusion du savoir ${ }^{20}$. Si les diffuseurs s'y refusent, alors leur légitimité à imposer des frais pour accéder au savoir peut être remise en cause.

19 Une autre possibilité, non discutée dans ces pages, consiste à faire payer la diffusion aux auteurs des articles (solution retenue par certaines revues, comme celles administrées par Springer, au travers de leur accès doré [Gold OA]). Cependant, une telle option a pour conséquence de marquer les inégalités matérielles entre chercheurs et institutions de recherche. De plus, des recherches prometteuses peuvent ne pas être diffusées par manque de fonds. Pour ces raisons, la possibilité peut être raisonnablement écartée pour le reste de la section.

20 On pourrait objecter que les chercheurs reçoivent déjà une compensation adéquate pour la production du savoir de la part des universités ou bailleurs de fonds. Toutefois, si un tel argument peut justifier de ne pas rétribuer les chercheurs, il ne justifie pas de ne pas payer pour l'usage du savoir par les diffuseurs, par exemple en rémunérant les universités ou les bailleurs de fonds qui ont financé la recherche en premier lieu. 
En vertu de la conception restrictive, l'utilisateur est celui qui consomme le produit final. Un tel argument pourrait alors justifier de faire payer aux usagers finaux (c'est-à-dire aux lecteurs, notion regroupant des individus et institutions) les coûts de diffusion et de recherche puisqu'ils ne tirent pas seulement avantage de la diffusion du savoir, mais également de sa production. Un problème évident est que l'accès au savoir peut se voir restreint par les ressources matérielles et financières des lecteurs. Une contribution des lecteurs finaux peut conduire à deux types d'inégalités: tout d'abord entre individus au sein d’un même pays, mettant à mal les principes d'égalité et d'inclusion entre citoyens, puis entre nations, conduisant à des asymétries de développement socio-économique.

Une seconde difficulté tient à l'identification des utilisateurs du savoir afin de les faire contribuer. De ce point de vue, l'existence des bénéfices distants pose problème. Socle des sociétés industrielles modernes, la mobilité et la fluidité du savoir rendent compliquée l’identification précise de l'ensemble des bénéficiaires. Le principe visant à faire contribuer telle entreprise ou tel individu pour l'usage d'une idée ou d'une avancée scientifique se heurte à la difficulté, surtout en l'absence d'un système de brevets, d’identifier les bénéficiaires de la diffusion de la recherche. Celle-ci est particulièrement prégnante dans la recherche en SHS.

Le second arrangement apparaît comme une solution à ces difficultés : il s'agit de faire payer l'ensemble des coûts (de recherche et de diffusion) par des fonds publics. Le principe n'est plus de faire payer la diffusion de la recherche par ses bénéficiaires, mais par l'État. Plusieurs raisons justifient un tel arrangement. En premier lieu, la nature de certains bénéfices sociaux fait qu'il devient possible d’attribuer à l'État un rôle central dans la diffusion du savoir. En effet, si l'on reconnaît l'importance du savoir pour la réalisation des idéaux d'inclusion et d'égalité, comment ne pas y voir une justification pour l'intervention directe de l'État?

Un tel argument trouve une résonnance particulière dans les sociétés industrielles fondées sur des principes démocratiques. En effet, l'idéal d'égalité se trouve au fondement même de l'architecture démocratique. Cela ne signifie nullement que cet idéal ne peut pas faire l'objet d'âpres discussions quant à son contenu et son interprétation. Mais s'il y a une conviction qui est partagée par les personnes de (presque) toute orientation politique, c'est celle qui stipule 
que les individus devraient bénéficier des mêmes opportunités dans l'existence, qu'ils devraient avoir une capacité (la plus large possible) de participer à la vie de leur société et qu'ils ne devraient pas souffrir d'exclusion. En outre, quelle que soit la conception que les citoyens aient de l'État, la plupart s'entendent pour lui attribuer la responsabilité morale de garantir inclusion et égalité.

Si l'État possède un devoir au regard des idéaux d'inclusion et d'égalité et si l'accès au savoir est un déterminant majeur pour la réalisation de ces idéaux, l'État a alors une responsabilité importante dans la diffusion du savoir. Celle-ci doit être d'en garantir l'accès sous des formes qui respectent les idéaux susmentionnés. Cependant, l'attribution d'un devoir à l'État n'équivaut nullement à justifier une forme particulière d'intervention, d'où l'émergence d'une question pratique : comment traduire cette responsabilité de l'État?

De nombreux arrangements sont possibles. L'État peut prendre en charge la diffusion de la recherche au travers de revues qu'ils financent. Outre sa lourdeur, un tel système suppose d'une part de renoncer à l'émulation concurrentielle entre différents diffuseurs et, d'autre part, d’accepter un degré assez conséquent d'ingérence. L'État peut aussi s'assurer que chaque individu a accès à un volume suffisant de savoir qui garantisse son inclusion ainsi que des opportunités égales sans prendre toutefois en charge lui-même cet accès. Il peut par exemple financer les diffuseurs ou leur imposer la gratuité de l'accès. Dans ce dernier cas, cela ne signifie pas que les diffuseurs perdent toute source de revenus. Ils peuvent transférer leurs coûts en amont, en demandant aux chercheurs, institutions de recherche ou bailleurs de fonds de payer pour la diffusion des recherches qu'ils entreprennent ou qu'ils financent. La différence avec le système actuel est que les lecteurs ne sont plus mis à contribution.

Cette dernière possibilité ressemble à une proposition récurrente dans les débats, à savoir faire payer les chercheurs pour la diffusion du savoir. C'est le principe du Gold Open Access ou accès libre «doré». Certains éditeurs, tels Springer, s'y sont déjà mis. Si l'on peut noter qu’un tel système garantit l'égalité et l'inclusion des citoyens dans l'économie du savoir, il génère d'autres inégalités, parmi les chercheurs. En fonction du prix demandé, il est possible que certains chercheurs ou institutions n'aient plus qu'une marge de manœuvre très 
réduite en termes de publications ${ }^{21}$. Pire, il est envisageable que des recherches d'une importance majeure ne puissent pas être publiées (et donc connues des pairs) pour des raisons budgétaires.

Quoi qu'il en soit, l'existence de bénéfices distants plaide en faveur d'une implication de l'État, sous une forme ou une autre. En effet, plutôt que d'essayer (vainement) d'identifier les bénéficiaires du savoir au risque de voir proliférer les cavaliers seuls ${ }^{22}$ (car des bénéficiaires saisissent l'opportunité de ne pas contribuer à la hauteur du bénéfice qu'ils retirent de la recherche), une alternative consiste à mutualiser les coûts de la diffusion du savoir. Puisque les bénéfices sont diffus, autant faire payer l'ensemble de la collectivité en se servant de l'État, seul agent capable d'imposer une contribution collective. L'argument est celui des biens publics: vu que l'on ne peut pas exclure les individus de la fourniture de certains biens, il devient préférable de faire contribuer tout le monde sur une base obligatoire (l'impôt). Des biens comme la police ou la défense nationale sont financés ainsi.

En résumé, il est trompeur de penser que la recherche puisse être d’accès libre dans le sens de gratuit. Quelqu'un doit payer pour sa diffusion, que de tels coûts soient pris en charge par les chercheurs, bailleurs de fonds, institutions de recherche ou l'État. Parmi ces options, l'engagement de l'État se trouve justifié pour plusieurs raisons. D’une part, l'État possède un certain nombre de responsabilités à l'égard des citoyens, notamment en matière d'inclusion et d'égalité. D’autre part, l'État possède des avantages indéniables dans la répartition des charges de la diffusion de la recherche.

21 Pour les revues éditées par Springer, ce prix tourne actuellement autour de 3000 US\$.

22 En théorie des jeux, un cavalier seul (ou free rider en anglais) est un agent qui profite d'un avantage offert par un autre agent sans y apporter sa contribution alors que celle-ci est requise. Le resquilleur des transports en commun ou le colocataire qui ne s'acquitte pas de sa part de ménage constituent des exemples de cavaliers seuls. 


\section{QUE PENSER DE LA RECOMMANDATION DE LA COMMISSION EUROPÉENNE?}

La Recommandation du I7 juillet 2012 de la CE se comprend à la lumière du questionnement sur la répartition des charges liées à la diffusion de la recherche ${ }^{23}$. Elle s'insère dans une perspective globale qui inclut des textes publiés par diverses organisations internationales en faveur de l'OA ${ }^{24}$. La Recommandation note que cet accès accru aux résultats de la recherche correspond à une évolution mondiale représentée par des textes d'autres institutions telles que l'UNESCO ou l'OCDE.

Son objectif est d'«assurer à la recherche financée par des fonds publics une large diffusion par la publication de données et d'articles scientifiques en libre accès ». Pour ce faire, la principale recommandation est de rendre les articles disponibles soit immédiatement à l'initiative du diffuseur, soit six mois après publication ( 12 mois dans le cas des SHS) au travers d'archives et à l'initiative de l'auteur. La CE envisage de dégager (ou faire dégager des fonds par les États) pour faciliter l'accès à la recherche, ce qui rejoint notre conclusion lors de la précédente section.

Quant aux motivations qui inspirent cette recommandation, Neelie Kroes, vice-présidente de la CE responsable de la stratégie numérique indique que «les contribuables ne devraient pas payer deux fois les travaux de recherche scientifique, et ils ont besoin d'un accès facile aux données brutes. Nous voulons améliorer radicalement la diffusion et l'exploitation des résultats de la recherche scientifique, car ces données constituent le nouvel or noir». Cette justification reflète la critique faite aux éditeurs anglophones qui constituent

23 Recommandation de la CE du 17 juillet 2012 relative à l'accès aux informations scientifiques et à leur conservation (Commission européenne, 2012).

24 Parmi les textes principaux qui marquent la montée en puissance de l'OA, figurent la Berlin Declaration on Open Access to Knowledge in the Sciences and Humanities (2003), la Déclaration sur l'accès aux données de la recherche financée par des fonds publics de l'OCDE (2004), le Projet révisé de stratégie relatif à la contribution de l'UNESCO à la promotion de l'accès libre à l'information et à la recherche scientifiques (201I) et Open Science for the $21^{\text {st }}$ century, A declaration of ALL European Academies (2012). 
un oligopole de profiter de l'entente qui existait entre éditeurs académiques et chercheurs il y a quelques décennies.

Pour sa part, Máire Geoghegan-Quinn, membre de la CE responsable de la recherche et de l'innovation, estime que «les contribuables doivent en avoir plus pour leur argent. Le libre accès aux publications et aux données scientifiques permettra à nos chercheurs et à nos entreprises de réaliser plus rapidement des avancées importantes, au bénéfice de la connaissance et de la compétitivité en Europe $\gg^{25}$. Cette prise de position souligne l'importance des bénéfices matériels et distants imputés à la recherche qui sont mis en exergue plus haut dans ce texte.

La Recommandation évoque cependant pêle-mêle différentes raisons qui relèvent de différentes logiques et s'adressent à différents acteurs. Parmi les raisons principales, elle considère que le libre accès aux résultats de la recherche facilitera la coopération, notamment à l'échelle européenne, permettra une meilleure intégration de la recherche aux besoins sociaux, améliorera la qualité de la recherche (en réduisant les doublons, c'est-à-dire les acteurs travaillant sur les mêmes thèmes de recherche, mais de manière séparée). Il est important de relever que les justifications avancées relèvent presque exclusivement des bénéfices matériels, négligeant les bénéfices socio-politiques et distants ; point sur lequel nous revenons ci-dessous.

La Recommandation arrive dans un contexte particulier qui voit la multiplication de donnés favorables à l'accès libre. Ce système produirait une variété d'avantages s'inscrivant dans la typologie des bénéfices sociaux évoqués plus haut. En guise d'illustration, John Houghton et Peter Sheehan (Houghton, Sheehan, 2009, p. I29-I30) estiment que le libre accès a des effets positifs sur la recherche (accélération du processus de découverte scientifique, réduction du risque de duplication de projets de recherche, maximisation des effets éducatifs, etc.), l'industrie et le gouvernement (gains de productivité, émergence de nouvelles industries, etc.) ainsi que la communauté dans son ensemble (consommateurs/citoyens mieux informés). 
Du point de vue des effets socio-politiques, l'OA favoriserait la démocratie, car tout le monde a accès à la recherche et peut la commenter. Plus fondamentalement, l'accès libre aux résultats de la recherche permet de réduire l'inégalité d’accès au savoir. Cet effet s'applique à la fois aux individus, mais également aux institutions de recherche (et bibliothèques). De la sorte, les disparités quant à l'accès au savoir sont réduites concernant nombre d’acteurs, par exemple entre pays développés et émergents.

Dans ce contexte, il faut reconnaître à la Recommandation le mérite d’avoir initié le débat sur le savoir, sa diffusion et la juste répartition des charges afférentes, en particulier en France ${ }^{26}$. Une lecture attentive du texte suscite toutefois un certain nombre d'interrogations, voire de réserves qui touchent à la fois aux limites de la Recommandation en tant que telle et de l'OA en général.

Tout d’abord, en rendant accessibles tous les articles de manière libre, le risque est fort d'inonder un marché de la publication scientifique qui montre déjà des signes de saturation (Collectif, 20I3c). Le nombre de publications scientifiques a crû de manière inquiétante, au point où de nombreux chercheurs avouent leurs difficultés à se tenir informés de l'actualité de leur domaine (Landes et al., 20I3a). L'accès libre à toutes les publications scientifiques va accroître cette difficulté à isoler l'information pertinente. Sur ce point précis, il s'agit du rôle dévolu aux revues établies, en général d’accès payant: trier le savoir en fonction de sa pertinence. Mais il est tout à fait envisageable qu'un processus de sélection se mette en place a posteriori, de manière plus ou moins coordonnée (par exemple, les États pourraient sélectionner les publications qui bénéficieront de leur soutien financier).

Ensuite, la Recommandation ne fait nulle mention des principes d'égalité et d'inclusion. Elle se concentre sur les bénéfices matériels de la recherche, en passant sous silence (ou presque) les bénéfices socio-politiques et distants. Si on laisse les seconds de côté, l'indifférence à l'égard des premiers déconnecte la Recommandation d'une conception plus large de la place du savoir dans les sociétés modernes. Dit autrement, si la Recommandation reconnaît la place que

26 Se reporter notamment à la motion «Open Access: le travail scientifique en sciences humaines et sociales et le débat public fragilisés par les mesures préconisées par la Commission européenne» ainsi qu'aux réactions suscitées par cette initiative (Collectif, 2013a, 2013b). 
le savoir joue dans l'amélioration des conditions matérielles des sociétés contemporaines, elle passe sous silence le fait que le savoir est tout aussi important dans la formation du citoyen moderne ainsi que dans la poursuite des idéaux d'égalité et d'inclusion. Sur cette question, elle s'éloigne des standards internationaux.

Il peut aussi lui être reproché de ne pas suffisamment prendre en compte la diversité des situations dans le monde de l'édition scientifique. En effet, à côté des mastodontes qui forment un oligopole dans l'univers de la publication anglophone, il y existe aussi une multitude d'éditeurs universitaires qui n'ont pas les taux de marge des premiers. De nombreuses revues survivent grâce à leurs abonnements et au travail gracieux des contributeurs et autres chercheurs. Cet aspect est particulièrement visible dans le cas français. Par conséquent, il est difficile de ne pas voir dans la Recommandation une réponse sur mesure aux dérives oligopolistiques que connaît l'édition anglophone (prix prohibitifs des abonnements, «Big Deal» qui oblige quasiment les bibliothèques et universités à s'abonner à des revues dont elles n’ont pas besoin, non-rémunération du travail des universitaires pour l'évaluation et la mise en forme du savoir, etc.). Mais, comme toute réponse sur-mesure, elle peut paraître être inadéquate, voire contre-productive pour les petits acteurs.

Enfin, la Recommandation peut nourrir l'illusion qu'il est possible de bénéficier d'un savoir gratuit, illusion renforcée par l'omniprésence des pratiques de piratage sur Internet. Mais aucun accès à la recherche ne peut être gratuit. La question n’est pas une question de gratuité ou non du savoir, mais procède plutôt de la détermination de l'instance qui est appelée à prendre à sa charge le coût de l'accès. Il est possible de reprocher à la Recommandation de ne pas suffisamment souligner la distinction entre l'accès et le financement. Établir le libre accès au savoir est une chose, déterminer les modalités de financement d'une telle diffusion en est une autre.

En définitive, la question touche aux bénéfices attendus de la diffusion du savoir. Il s’agit aussi d’une question empirique qui doit être évaluée comme telle, c'est-à-dire au travers d'études d'impact. [Pour une analyse plus étoffée de cette question, se reporter à l'article de Ghislaine Chartron publié dans ce dossier.] La question est primordiale puisque lorsque des propositions, comme celles de la Recommandation, sont évaluées, elles doivent l'être au regard des bénéfices que la société est en droit d'attendre de la recherche et de sa diffusion. De ce point de vue, force est de 
constater que si des études tendent à confirmer les bénéfices qui pourraient être produits par l'adoption de l'accès libre pour la recherche financée par des fonds publics (Houghton, Sheehan, 2009; Houghton, Oppenheim, 2010), d'autres études sont beaucoup plus sceptiques (Hall, 20ı) Enfin, notons que l'évaluation des bénéfices doit également prendre en compte l'impact sur l'interdisciplinarité de la recherche. [Le sujet est abordé par Hans Dillaerts dans l'article qu'il publie dans ce dossier.]

\section{CONCLUSION Les enjeux normatifs et politiques de l'Open Access}

La conclusion se borne à dresser un rapide état des lieux des questions que la Recommandation laisse en suspens, mais qui apparaissent centrales dans le problème de la répartition des charges de la recherche. Les enjeux normatifs et politiques mis à jour par la Recommandation et, plus généralement, le débat relatif à la diffusion du savoir couvrent trois thèmes.

Tout d’abord, il est impossible de trop insister: la communauté académique fait actuellement l'expérience d'un problème sérieux qui est celui de la course à la publication. Du fait d'une évaluation de la qualité de la recherche en termes principalement quantitatifs (nombre d’articles publiés), même si le qualitatif (prestige de la revue) n'est pas totalement absent, les chercheurs se trouvent dans l'obligation de publier toujours plus afin d'avoir une chance d'obtenir un poste ou des fonds de recherche. La compétition peut avoir du bon, mais l'excès de compétition peut tout aussi bien se révéler dévastateur, ce qui est en l'occurrence le cas. La course à la publication a des effets délétères sur le bien-être des chercheurs ${ }^{27}$ ainsi que sur la qualité de la recherche puisque plus de publications a souvent comme corollaire une qualité moindre.

En dépit de son importance, la Recommandation passe sous silence le problème de l’accès aux fruits de la recherche. En résumé, un des défis de la diffusion de la recherche est très certainement de mettre un frein à l'accroissement du nombre de publications afin d’en garantir la qualité ainsi que le traite-

27 Une étude menée au Royaume-Uni montre que ces derniers ont des niveaux de stress et de détresse psychologique très élevés (Kinman et al., 2006). 
ment par les chercheurs concernés. En d’autres termes, le débat sur l'OA est le moment idéal pour ouvrir celui sur les conditions d'efficacité de la recherche.

Ensuite, la deuxième série d’enjeux est de type démocratique. Ces derniers font écho à l'importance socio-politique de la diffusion de la recherche. Il est nécessaire de remarquer que l'égalité d’accès ne suffit pas. Des individus peuvent avoir accès au même volume de savoir (comme dans le cas de l'OA) sans bénéficier des mêmes capacités de traitement de l'information. Dès lors, si l'on considère que le rôle du savoir et de sa diffusion est en partie de former le citoyen moderne, c'est-à-dire un individu capable de prendre des décisions éclairées quant à sa vie et sa communauté, alors il est impossible de ne pas s'interroger sérieusement sur les capacités à profiter d'une diffusion libre du savoir. Cette question doit faire également l'objet d'une discussion dans le cadre de la Recommandation au travers d'institutions qui facilitent la compréhension et l'intégration du savoir.

Enfin, une question demeure centrale : celle des effets distants. Les bénéfices de la recherche ne se limitent pas aux applications industrielles et commerciales immédiates, pas plus qu'aux effets socio-politiques. Le savoir infuse littéralement la société. Il se répand parmi les individus et institutions pour produire des changements, plus ou moins marqués. L'importance de la recherche en SHS pour la stabilité et la vitalité des institutions démocratiques a déjà été mentionnée. De ce point de vue, il y a un manque cruel de concepts et de données susceptibles de contribuer à une identification plus fine des bénéficiaires et des retombées distants de la recherche. Plus encore, il y a un défaut criant d'une articulation de ces concepts et données afin de présenter une théorie de l'influence de la diffusion du savoir sur le fonctionnement démocratique de nos sociétés. Il s'agit d’ailleurs peut-être de l'enjeu ultime derrière l'OA: un choix de société authentiquement moderne, c'est-à-dire portant sur la gestion de l'information.

Remerciements: Cet article a bénéficié des précieux conseils de David Budtz Pedersen ainsi que des commentaires de Martin Marchman et Morten Nielsen. 


\section{BIBLIOGRAPHIE}

ABERGANTI Michel, 20I3, «Économie de la publication scientifique et libre accès: un débat relancé par la mort d'Aaron Swartz», Slate, 21 janvier: < http://www.slate.fr/ story/67263/suicide-aaron-swartz-economie-publication-scientifique-libre-acces>.

COLLECTIF, 20I3a, «Open Access: le travail scientifique en sciences humaines et sociales et le débat public fragilisés par les mesures préconisées par la Commission européenne», Paris: <http://www.openaccess-shs.info/motion/>.

-, 20I3b, «Qui a peur de l'Open Access», Le Monde, 15 mars: <http://lemonde.fr/ sciences/article/20|3/03//5/qui-a-peur-de--open-acces_|848930_|650684.html>.

-, 2013c, «La science menacée par une bulle spéculative de l'édition?», Le Monde,

II novembre: <http://lemonde.fr/sciences/article/2013//I/I//a-science-menaceepar-une-bulle-speculative-de-I-edition_35||86|_|650684.html>.

COMMISSION EUROPÉENNE, 2012, Recommandation de la Commission du 17.27.2012 relative à l'accès aux informations scientifiques et à leur conservation, Bruxelles: $<$ http://ec.europa.eu/research/science-society/document_library/pdf_06/ recommendation-access-and-preservation-scientific-information_fr.pdf>.

BATTAGLIA Mattea, COLLAS Aurélie, 2013, «Classement PISA: la France championne des inégalités scolaires», Le Monde, 3 décembre: <http://lemonde.fr/ ecole-primaire-et-secondaire/article/20 /3/I2/03/classement-pisa-la-francechampionne-des-inegalites-scolaires_3524389_|473688.html>.

BOURDIEU Pierre, 1979, La Distinction, Paris, Éditions de Minuit.

-, PASSERON Jean-Claude, 1970, La Reproduction: Éléments pour une théorie du système d'enseignement, Paris, Éditions de Minuit.

COHEN Daniel, 2006, Trois leçons sur la société post-industrielle, Paris, Seuil.

CONLEY John P., WOODERS Myrna, 2009, «But what have you done for me lately? Commercial Publishing, Scholarly Communication, and Open-Access », Economic Analysis and Policy, 39-1, p.7I-87.

COUR DES COMPTES, 2013, Le Financement public de la recherche, un enjeu national, Rapport public thématique, Paris: <http://www.ccomptes.fr/Publications/ Publications/Le-financement-public-de-la-recherche-un-enjeu-national>. 
EDLIN Aaron S., RUBINFELD Daniel L., 2005, «The Bundling of Academic Journals», American Economic Review, 95-2, p.441-446.

HALL Steven, 2010, «A commentary on "The economic implications of alternative publishing models" », Prometheus, 28-I, p.73-84.

HEATH Joseph, 2002 (200I), La Société efficiente: Pourquoi fait-il si bon vivre au Canada? Montréal, Les Presses de l'Université de Montréal.

-, 2006, «The Benefits of Cooperation», Philosophy and Public Affairs, 34-4, p.313-35I.

HOUGHTON John, OPPENHEIM Charles, 2010, «The economic implications of alternative publishing models », Prometheus, 28-I, p. 4l-54.

HOUGHTON John, SHEEHAN Peter, 2009, «Estimating the Potential Impacts of Open Access to Research Findings», Economic Analysis and Policy, 39-I, p. I27-I42.

KINMAN Gail, JONES Fiona, KINMAN Russell, 2006, «The Well-being of the UK Academy, 1998-2004», Quality in Higher Education, 12-I, p. I5-27.

LANDES Xavier, MARCHMAN Martin, NIELSEN Morten, 201 I, «Les Chercheurs sont prisonniers d'une course à la publication », Le Monde, 21 janvier:

<http://abonnes.lemonde.fr/idees/article/201 I/01/21/les-chercheurs-sontprisonniers-d-une-course-a-la-publication_|468|55_3232.html>.

-, 2013a, «The Academic Rat Race: Dilemmas and Problems in the Structure of Academic Competition », Learning and Teaching: The International Journal of Higher Education in the Social Sciences (LATISS), 5-2, p. 73-90.

-, 2013b, «Publications académiques: Le règne de l'absurde», Rue89, 2 janvier: <http://rue89.nouvelobs.com/2013/01/02/ publications-academiques-le-regne-de-labsurde-238234>.

MÉDA Dominique, 2008, Au-delà du PIB: Pour une autre mesure de la richesse, Paris, Flammarion.

NEWFIELD Christopher, 20II, Unmaking the Public University: The Forty-Year Assault on the Middle Class, Cambridge, Harvard University Press.

PEDERSEN David Budtz, HENDRICKS Vincent F., 2013, «Science Bubbles», Philosophy \& Technology, p. I-16. 
PEDERSEN David Budtz, STJERNFELT Frederik, 20I4, «The Social Epistemology of Open Access 》, Humanomics, I-2, p. I- 12.

PERSSON Karl Gunnar, 2010, An Economic History of Europe: Knowledge, Institutions and Growth, 600 to Present, New York, Cambridge University Press.

POLLITT Christopher, 1993, Managerialism and the Public Services, Oxford, Blackwell.

SALTER Ammon J., MARTIN Ben R., 200I, «The Economic Benefits of Publicly Funded Basic Research: A Critical Review», Research Policy, 30-3, p. 509-532.

SCHUMPETER Joseph, 1990 (1942), Capitalisme, socialisme et démocratie, Paris, Payot.

The Chronicle of Higher Education, 2012, «Want to Change Academic Publishing? Just Say No», September 23.

WEILER Hans N., 2000, «States, Markets and University Funding: New Paradigms for the Reform of Higher Education in Europe», Compare: A Journal of Comparative and International Education, 30-3, p. 333-339. 